\title{
INFECÇÃO FÚNGICA NO TRANSPLANTE DE ÓRGÃOS ABDOMINAIS, COM FOCO EM INTESTINAL E MULTIVISCERAL - REVISÃO DA LITERATURA
}

\author{
Fungal infection aiming Small Bowel and Multivisceral Transplantation - \\ Literature Review
}

\author{
Adriana Weinfeld Massaia ${ }^{1}$, André Ibrahim David ${ }^{2}$, Catiana Mitica Gritti ${ }^{3}$, Ivan Leonardo Avelino França e Silva ${ }^{4}$, Edson Abdala ${ }^{5}$
}

\section{RESUMO}

A natureza das infecções após os transplantes de órgãos sólidos mudou, significativamente, com o advento de potentes esquemas imunossupressores, o uso rotineiro de profilaxia antimicrobiana e os avanços das técnicas de diagnóstico microbiológico. Novos patógenos estão sendo identificados nessa população, incluindo vários com significante perfil de resistência a antimicrobianos. Cada tipo de enxerto apresenta riscos de infecções particulares, sendo os transplantes de intestino e multivisceral importantes exemplos disso. Entretanto, os padrões gerais de infecção são similares a todos os transplantes de órgãos sólidos. As infecções nessas situações podem ser divididas em categorias: relacionadas ao doador, relacionadas ao receptor, infecções adquiridas na comunidade e infecções relacionadas à assistência à saúde. A cronologia das infecções após transplante é determinada pela natureza e intensidade das exposições epidemiológicas individuais e do estado de equilíbrio da imunossupressão. A escassez de estudos epidemiológicos das infecções pós-transplantes de intestino e multivisceral ainda mantêm sua compreensão, mais no estado da arte do que como ciência. O objetivo deste estudo é descrever os dados epidemiológicos das infecções fúngicas pós-transplantes de intestino e multivisceral. Os agentes mais prevalentes nos transplantes de intestino e multivisceral foram: Candida spp. e Aspergillus spp. Os principais sítios de infecção foram: sítio cirúrgico, intra-abdominal, corrente sanguínea, cateter venoso central, urinário e respiratório. Os fatores de risco identificados foram: rejeição aguda, dose alta de imunossupressão, uso de dispositivos invasivos, cirurgias extensas e reoperação, enxerto contaminado, infecção prévia e paciente hospitalizado no período pré-transplante.

Descritores: Infecção; Micose; Transplante; Intestino Delgado.

Instituições:

${ }^{1}$ Departamento de Infectologia da Santa Casa de São Paulo, São Paulo/SP

${ }^{2}$ Unidade de Transplante de Fígado da Beneficência Portuguesa de São Paulo, São Paulo/SP

${ }^{3}$ Gestão de Transplante de Fígado da Beneficência Portuguesa de São Paulo, São Paulo/SP

${ }^{4}$ Departamento de Infectologia e SCIH do Hospital A.C.Camargo, São Paulo/SP

${ }^{5}$ Departamento de Infectologia do Hospital das Clinicas da FMUSP, São Paulo/SP

\section{Correspondência:}

Nome: Adriana Weinfeld Massaia

Endereço: Rua Itapirapuã, 278 - São Paulo/SP

Tel: (11) 98199-7378

E-mail: dri.weinfeld@gmail.com

\section{INTRODUÇÃO}

Pacientes submetidos a Transplante de Intestino (TI) são mais suscetíveis a infecções do que qualquer outro receptor de Transplante de Órgãos Sólidos (TOS). ${ }^{1}$ O estado de equilíbrio da imunossupressão é um conceito utilizado para descrever os fatores que podem contribuir para o risco de infecção individual. Esse risco é amplamente determinado pela dose, duração e sequência da terapia imunossupressora. No entanto, coleções pós-operatórias, uso de dispositivos invasivos, uso de drenos, isquemia tecidual, neutropenia, doenças crônicas associadas, disfunção orgânica, distúrbios metabólicos, exposição antimicrobiana, entre outros, também contribuem para o risco de adquirir infecção. ${ }^{2,3}$ Os fatores de risco individuais devem ser considerados para escolha de estratégia profilática e terapia empírica, e para determinação dos diagnósticos diferenciais de cada infecção. ${ }^{2-4}$ 
A incidência de infecção fúngica invasiva (IFI) nos TOS depende do órgão transplantado, da terapia imunossupressora e de complicações cirúrgicas, variando de 2 a $60 \%$. A maioria dessas infecções é causada por espécies de Candida, provenientes de sítio endógeno, e de Aspergillus.

Porém, são também descritas infecções por espécies de Cryptococcus e por Pneumocystis jirovecii. ${ }^{4,5}$

\section{OBJETIVO}

Descrever os dados epidemiológicos das infecções fúngicas pós-transplantes de intestino e multivisceral quanto a incidência, distribuição, fatores de risco e evolução, segundo revisão da literatura.

\section{MATERIAL E MÉTODOS}

Foi realizada revisão de literatura através de busca no Medline/PubMed, Latin-American and Caribbean Centre on Health Sciences Information (Lilacs), Scientific Electronic Library Online (Scielo), Embase Biomedica Answers, Thomson Reuters (ISI) Web of Knowledge e SciVerseScopus. Os termos utilizados foram: "intestine transplantation", "intestine transplant", "intestinal transplantation", "intestinal transplant", "multivisceral transplantation", "small bowel transplantation", "infectious transplantation", "intest* transplant", "small bowel transplant" "multivisceral transplant", "infection transplantation", "bacterial infection transplant", "viral infection transplant*", "fungal infection transplant*". Não se utilizou filtro. O período de revisão foi a partir do ano de 2000 até julho de 2013.

Os seguintes artigos foram selecionados para compor os dados de revisão:

A. Artigos originais que tratavam de TI ou TMV, e uma das seguintes situações: descrições de casuísticas com resultados e complicações, incluindo infecciosas; relatos ou estudos de infecções.

B Artigos de revisão sobre TI ou TMV que incluíam informação sobre infecção.

C. Artigos originais ou de revisão em TOS que contivessem dados de infecção em TI ou TMV.

\section{ANÁLISE DE RESULTADOS}

Nos TI e Transplantes Multiviscerais (TMV) os agentes predominantes são espécies de Candida e Aspergillus (Tabela 1). ${ }^{4-9}$
Tabela 1 - Infecções fúngicas nos transplantes de intestino e multivisceral

\begin{tabular}{|c|c|c|}
\hline Autor / Origem / Ano & $\begin{array}{c}\text { Número total de } \\
\text { pacientes / Número } \\
\text { total de episódios } \\
\text { de infecção }\end{array}$ & Agentes $\dagger$ \\
\hline $\begin{array}{c}\text { Akhter K et al/ } \\
\text { Washington/ } 2012^{10}\end{array}$ & $56 / 85^{*}$ & $\begin{array}{c}\text { 2/85 (2,3\%) } \\
\text { C.albicans: } 1 \\
\text { C.parapsilosis: } 1\end{array}$ \\
\hline $\begin{array}{l}\text { Florescu DF et al/ } \\
\text { Nebraska/ } 2012^{11}\end{array}$ & $98 / 146^{*}$ & $\begin{array}{l}\text { 14/146 (9,5\%) } \\
\text { C.albicans: } 4 \\
\text { C. glabrata: } 4 \\
\text { C.parapsilosis: } 4 \\
\text { C.humicolus: } 1 \\
\text { Candida spp: } 1\end{array}$ \\
\hline $\begin{array}{l}\text { Florescu DF et al/ } \\
\text { Nebraska/ } 2010^{12}\end{array}$ & $98 / 63^{* *}$ & $\begin{array}{c}\text { 63/63 (100\%) } \\
\text { C.albicans: } 22 \\
\text { C. glabrata: } 15 \\
\text { C.parapsilosis: } 8 \\
\text { C.krusei: } 4 \\
\text { C.tropicalis: } 3 \\
\text { C.lamblia: } 1 \\
\text { Aspegillus spp.: } 3\end{array}$ \\
\hline $\begin{array}{l}\text { Rosado-Odom VM et } \\
\text { al/ Indiana/ } 2011^{13}\end{array}$ & $121 / 533^{* * *}$ & $\begin{array}{c}71 / 533 \text { (13,3\%) } \\
\text { C.glabrata: } 39 \\
\text { C.krusei: } 11 \\
\text { C.albicans: } 10 \\
\text { C.parapsilosis: } 5 \\
\text { C.lusitaniae: } 3 \\
\text { R.mucilaginosa: } 1 \\
\text { A.fumigatus } 1 \\
\text { Ductylaria spp.: } 1\end{array}$ \\
\hline $\begin{array}{l}\text { Trentadue J et al/ } \\
\text { Argentina/ } 2011^{14}\end{array}$ & $15 / \mathrm{ND}^{\star * *}$ & $\begin{array}{c}N D \\
\text { Candida spp.: } 1 \\
\text { Cryptococcus spp.: } 1\end{array}$ \\
\hline $\begin{array}{l}\text { John M et al. / Nova } \\
\text { Iorque/ } 2006^{15}\end{array}$ & $31 / 42^{* * *}$ & $\begin{array}{l}\text { 5/42 (12\%) } \\
\text { C.albicans: } 1 \\
\text { C.parapsilosis: } 1 \\
\text { C.tropicalis: } 1 \\
\text { C.glabrata: } 1 \\
\text { A.fumigatus: } 1\end{array}$ \\
\hline $\begin{array}{l}\text { Guaraldi G et al/ } \\
\text { Itália/ } 2005^{16}\end{array}$ & $19 / 100^{* * *}$ & $\begin{array}{c}\text { 6/100 (6\%) } \\
\text { C.albicans: } 3 \\
\text { A.fumigatus: } 2 \\
\text { C.krusei: } 1\end{array}$ \\
\hline
\end{tabular}

† Um paciente pode ter apresentado mais de um episódio de infecção

* Infecção de corrente sanguínea

** Infecção exclusivamente fúngica

*** Infecções por agentes e em locais variados

As espécies de Candida são responsáveis por mais de $50 \%$ das IFI em pacientes submetidos a TOS. Ela ocorre precocemente quando comparada a outros fungos, geralmente nos primeiros três meses após o transplante, como infecção nosocomial. Os principais sítios são a corrente sanguínea e o trato gastro-intestinal (TGI). Os fatores de risco relacionados são: idade, terapia antimicrobiana de amplo espectro, dispositivos invasivos, 
terapia parenteral, neutropenia prolongada, permanência em unidade de terapia intensiva, imunossupressão e terapia de substituição renal. ${ }^{1,4,8,9}$

Um estudo prospectivo analisou a epidemiologia e o desfecho de IFI em 429 TOS. Candidíase invasiva foi diagnosticada em $59 \%$ dos casos, e a C.albicans foi o agente isolado mais comum $(46,4 \%)$. Os pacientes que receberam profilaxia antifúngica tiveram mais infecção por C.glabrata e Candida krusei (C.krusei). Os sítios de infecção encontrados foram corrente sanguínea (53\%), intra-abdominal $(36,8 \%)$, pulmão $(10,2 \%)$ e pele $(4,1 \%)$. Aspergillus foi o segundo patógeno mais frequente $(24,8 \%)$. Desses pacientes estudados, 12 foram submetidos a $\mathrm{TI}$ e todos receberam profilaxia antifúngica. ${ }^{9,12,17}$

Nos TI e TMV, IFI foi identificada em 40 a 59\%, ocasionada principalmente pela ruptura da integridade do TGI, alta imunossupressão e elevada incidência de infecção por CMV. O período de maior risco foi igual aos demais TOS, no entanto, um número considerável de infecção por Candida ocorreu além desse período de risco. ${ }^{1,4,8,18}$

Florescu D. e colaboradores realizaram estudo retrospectivo tipo caso-controle com 46 pacientes pediátricos, avaliando os fatores de risco para infecção por Candida nos TI e TMV. Foram encontrados 40 episódios de infecção por Candida spp., 20 na corrente sanguínea, 15 intra-abdominais, três envolvendo múltiplos sítios e um empiema. Os fatores de risco associados foram uso de nutrição parenteral total (NPT) uma semana antes e uso de antibióticos sete dias antes da infecção. ${ }^{17}$

Esses mesmos autores publicaram outro estudo com 98 pacientes também pediátricos que receberam 110 TI ou TMV. Destes, 25 desenvolveram 59 episódios de infecção por Candida e quatro por Aspergillus. Das infecções por Candida, 39 apresentaram fungemia, 17 infecções intra-abdominais, duas infecções do trato urinário e uma empiema. C.albicans foi o agente isolado mais frequente, responsável por $37,3 \%$ dos casos, e C.glabrata por $25,4 \%$, C.parapsilosis $13,3 \%$, C. krusei $6,8 \%$, Candida tropicalis (C.tropicalis) $5,1 \%$ e Candida lambia (C.lambia) 1,7\%. Em relação ao tempo de diagnóstico da infecção após o transplante, 11 casos ocorreram até o primeiro mês e 12 entre o primeiro e o sexto mês, sendo o restante após seis meses. Descrição das espécies de Candida por sítio de infecção e tempo pós-transplante deste estudo encontra-se na Tabela 2.

Os quatro episódios de infecção por Aspergillus foram de doença invasiva em TMV. Trinta pacientes evoluíram a óbito, e aspergilose invasiva foi fator de risco para mortalidade na análise multivariada. ${ }^{6,12}$
Tabela 2 - Linha do tempo das infecções após transplante de intestino e multivisceral segundo espécie de Candida e sítio de infecção. ${ }^{12}$

\begin{tabular}{|c|c|c|}
\hline $\begin{array}{l}\text { Tempo após } \\
\text { transplante }\end{array}$ & $\begin{array}{c}\text { Sítio de infecção } \\
\text { (número de infecção) }\end{array}$ & $\begin{array}{c}\text { Espécie de Candida } \\
\text { (número) }\end{array}$ \\
\hline 0-1 mês & $\begin{array}{l}\text { Infecção Abdominal (IA) (7) } \\
\text { Infecção de corrente } \\
\text { sanguínea (ICS) (3) } \\
\text { Pleura (1) }\end{array}$ & $\begin{array}{l}\text { C.albicans (2) } \\
\text { C.glabrata (2) } \\
\text { C.krusei (2) } \\
\text { Miscelânea (1) } \\
\text { C.glabrata (2) } \\
\text { C.parapsilosis (1) } \\
\text { C.glabrata (1) }\end{array}$ \\
\hline 1-6 meses & $\begin{array}{c}\text { IA (6) } \\
\text { ICS (5) } \\
\text { Infecção do trato-urinário } \\
\text { (ITU) (1) }\end{array}$ & $\begin{array}{c}\text { C.albicans (3) } \\
\text { C.glabrata (1) } \\
\text { C.krusei (1) } \\
\text { Miscelânea (1) } \\
\\
\text { C.albicans (1) } \\
\text { C.parapsilosis (2) } \\
\text { C.tropicalis (2) } \\
\text { C.albicans (1) }\end{array}$ \\
\hline$>6$ meses & $\begin{array}{l}\text { ICS (31) } \\
\text { ITU (1) }\end{array}$ & $\begin{array}{l}\text { C.albicans (2) } \\
\text { C.glabrata (2) } \\
\\
\text { C.albicans (12) } \\
\text { C.glabrata (7) } \\
\text { C.krusei (1) } \\
\text { C.parapsilosis (5) } \\
\text { C.tropicalis (1) } \\
\text { C.lambica (1) } \\
\text { Miscelânea (4) } \\
\\
\text { C.krusei (1) }\end{array}$ \\
\hline
\end{tabular}

Aspergilose invasiva é a segunda causa mais importante de IFI nos TOS, com taxa entre 1 e $15 \%$. Dados iniciais reportaram mortalidade de mais de $65 \%$, sendo quase $100 \%$ se não tratados, no entanto atualmente esta se aproxima de $22 \%$. Os fatores de risco descritos são retransplante, falência renal com necessidade de terapia de substituição, outras cirurgias e infecção por CMV. Nos TI e TMV, essa doença representa 1 a $3 \%$ das IFI, com mortalidade associada de 60 a $90 \%$, porém esses dados foram obtidos de publicações com pequeno número de casos. Não há trabalhos que descrevam fatores de riscos específicos em TI e TMV. ${ }^{1,4,6,8,18,19}$

Criptococose acomete 0,3 a $5,3 \%$ dos receptores de TOS, com mortalidade de $20 \%$. Aproximadamente 25 a $54 \%$ desses receptores apresentam doença pulmonar, e a infecção disseminada, incluindo sistema nervoso central e fungemia, ocorrem em 52 a $61 \%$ e 20 a $25 \%$ dos receptores de TOS, respectivamente. Na maioria dos casos, a doença é considerada reativação de infecção quiescente, entretanto há relatos de infecção primária, especialmente transmitida pelo doador. O tempo entre o transplante e a infecção varia de 16 a 21 meses, e 
quando ocorre precocemente, nos primeiros 30 dias após o transplante, deve-se aventar a possibilidade de transmissão pelo enxerto. O uso de inibidores de calcineurina como agentes imunossupressores parece estar associado a menor incidência de doença disseminada e, consequentemente, relacionar-se a doença limitada ao pulmão. De um modo geral, os fatores de risco envolvidos são: uso de corticosteróide, pacientes cirróticos e uso de alemtuzumab, principalmente como tratamento contra rejeição celular. ${ }^{20-22}$

O estudo TRANSNET, realizado com 15 centros dos EUA, corrobora esses dados sobre IFI. Durante o período estudado, 1208 IFI ocorreram em 1063 receptores de órgão sólido, sendo $17 \mathrm{TI}$ e TMV. As IFI identificadas foram: candidíase $53 \%$, aspergilose $19 \%$, criptococose $8 \%$, fungos filamentosos não-Aspergillus $8 \%$ e fungos endêmicos $5 \%$. Entre os pacientes submetidos a TI ou TMV, foram observados 22 episódios de IFI, e os agentes responsáveis foram Candida spp. em 19 (85\%), Cryptococcus spp. em um (5\%), outras leveduras e não identificados em dois (10\%). ${ }^{23} \mathrm{O}$ mesmo foi observado em uma análise na Argentina, que evidenciou um caso de criptococose entre os 15 pacientes submetidos a TI. ${ }^{14}$

A histoplasmose pode causar infecção em receptores de TOS através da reativação de foco latente, como infecção primária ou por transmissão do doador. Não há dados na literatura que permitam definir sobre a incidência desta infecção nos TI e TMV. Um estudo retrospectivo analisou os TOS (não inclui TI ou TMV) de 1997 a 2007 em área endêmica. Observou 29 casos de histoplasmose em 3436 TOS, sendo que 14 eram ativos confirmados, 10 tiveram confirmação no órgão explantado, quatro apresentaram evidência no tecido do doador e um teve histoplasmose antes do transplante. ${ }^{24}$ No Brasil, observou-se ocorrência de histoplamose em três de $82(0,2 \%)$ casos de infecção endêmica oportunista, entre pacientes submetidos a transplante de rim ou fígado. ${ }^{25}$

Com o advento da profilaxia e esquemas imunossupressores com doses reduzidas, as infecções por Pneumocystis jirovecii diminuíram. Dados reportam incidência de 5 a 15\% em TOS, previamente à introdução da profilaxia, e não há relato recente dessa complicação nos TI e TMV.6,26

\section{CONCLUSÃO}

Os agentes mais prevalentes nos transplantes de intestino e multivisceral foram: Candida spp. e Aspergillus spp. Os principais sítios de infecção foram: sítio cirúrgico, intraabdominal, corrente sanguínea, cateter venoso central, urinário e respiratório. Os fatores de risco identificados foram: rejeição aguda, dose alta de imunossupressão, uso de dispositivos invasivos, cirurgias extensas e reoperação, enxerto contaminado, infecção prévia e paciente hospitalizado no período pré-transplante.

\section{ABSTRACT}

The nature of infections after solid organ transplantation has changed significantly with the advent of potent immunosuppressive regimens, the routine use of antibiotic prophylaxis and the advancement of microbiological diagnostic techniques. New pathogens are being identified among this population, including several with significant antimicrobial resistance profile. Each type of graft has specific risks for infections, and both intestine and multivisceral transplants are important examples. However, the general infection patterns are similar to all solid organ transplants. Infections in these situations can be divided into categories: related to the donor, related to the receiver, infections acquired in the community and infections related to health care. The post-transplant chronology of infections is determined by the nature and intensity of individual epidemiological exposure and balance in the immunosuppression state. The scarcity of epidemiological studies on bowel and multivisceral post-transplant infections still maintain their understanding more as a state of the art than as a science. The purpose of this study is to describe the epidemiological data of the intestine and multivisceral post-transplant fungal infections. The most prevalent agents in the intestine transplants and multivisceral were: Candida spp. and Aspergillus spp. The main spots of infection were the surgical site, intra-abdominal, blood, central venous catheter, urinary and respiratory systems. The identified risk factors were: acute rejection, high dose of immunosuppression, use of invasive devices, extensive surgery and reoperation, contaminated graft, previous infection and hospitalized patients in the pre-transplant period.

Keywords: Infection; Mycoses; Transplantation; Intestine, Small. 


\section{REFERÊNCIAS}

1. Palocaren MS. An overview of intestine and multivisceral transplantation. Crit Care Nurs Clin Norht Am. 2011;23:457-69.

2. Fishman JA, Issa NC. Infection in organ transplantation: risk factors and evolving patterns of infection. Infect Dis Clin North Am. 2010;24:273-83.

3. Fishman JA and the AST Infectious Diseases Community of Practice. Introduction: infection in solid organ transplant recipients. Am J Transpl. 2009;9(Suppl 4):S3-S6

4. Muñoz P, Fernández NS, Fariñas MC. Epidemiology and risk factors of infections after solid organ transplantation. Enferm Infecc Microbiol Clin. 2012;30(Supl 2):10-8.

5. Palocaren MS. An overview of intestine and multivisceral transplantation. Crit Care Nurs Clin Norht Am. 2011;23:457-69.

6. Timpone Jr JG, Girlanda R, Rudolph L, Fishbein TM. Infections in intestinal and multivisceral transplant recipients. Infect Dis Clin North Am. 2013;27:359-77.

7. Fishman JA. Infection in solid organ transplant recipients. $\mathrm{N}$ Engl J Med. 2007;357:2601-14.

8. Grim SA, Clark Nm. Management of infectious complications in solid organ transplant recipients. Clin Pharmacol Ther. 2011;90:333-42

9. Silveira FP, Kusne S; AST Infectious Diseases Community of Practice. Candida infections in solid organ transplantation. Am J Transpl. 2013;13:220-7

10. Akhter K, Timpone J, Matsumoto C, Fishbein T, Kaufman S, Kumar P. Six-month incidence of bloodstream infections in intestinal transplant patients. Transpl Infect Dis. 2012;14:242-7

11. Florescu DF. Qiu F, Langnas AN, Mercer DF, Chambers $\mathrm{H}$, Hill LA, Qaragholi N, Kalil AC. Bloodstream infections during the first year after pediatric small bowel transplantation. Pediatr Infect Dis J. 2012;31:700-4.

12. Florescu DF, Islam KM, Grant W, Mercer DF, Langnas A, Botha J, et al. Incidence and outcome of fungal infections in pediatric small bowel transplant recipients. Transpl Infect Dis. 2010;12:497-504

13. Rosado-Odom VM, Johnson R, Mangus RS. Bacterial, fungal, and parasitic infections one year after isolated intestinal and multivisceral transplantation in a single centersession: Poster Abstract Session: Infections after solid organ transplants. Annual Meeting IDSA; Boston; 2011.

14. Trentadue J, Rumbo C, Hervás MDG, Saá G, Martínez MI, Orce G, et al. Transplante intestinal en pediatria. Análisis de la primera serie de receptores en la Argentina. Arch Argent Pediatr 2011;109(2):135-141

15. John M, Gondolesi G, Herold BC, Kaufman S, Fishbein T, Posada R. Impact of surveillance stool culture guided selection of antibiotics in the management of pediatric small bowel transplant recipients. Pediatr Transpl. 2006:10:198-204
16. Guaraldi G, Cocchi S, Codeluppi M, Di Benedetto F, De Ruvo N, Masetti M, et al. Outcome, incidence and timing of infectious complications in small bowel and multivisceral organ transplantation patients. Transplantation. 2005;80:1742-8

17. Florescu DF, Quu F, Mercer DF, Langnas AN, Shafer LR, Kalil AC. Risk factors for systemic candida infections in pediatric small bowel transplant recipients. Pediatr Infect Dis J. 2012;31:120-3.

18. Neofytos D, Fishman JA, Horn D, Anaissie E, Chang CH, Olyaei A, et al. Epidemiology and outcome of invasive fungal infections in solid organ transplant recipients. Transpl Infect Dis. 2010:12:220-9

19. Vianna R, Misra V, Fridell JA, Goldman M, Mangus RS, Tector J. Survival after disseminated invasive aspergillosis in a multivisceral transplant recipient. Transpl Proc. 2007;39:305-7

20. Sun HS, Alexander BD, Lortholary O, Dromer F, Forrest GN, Lyon GM, et al.; Cryptococcal Collaborative Transplant Study Group. Unrecognized pretransplant and donorderived cryptococcal disease in organ transplant recipients. Clin Infect Dis. 2010;51:1062-9

21. Singh N, Alexander BD, Lortholary O, Dromer F, Gupta KL, John GT, et al. Pulmonary criptococcosis in solid organ transplant recipients: clinical relevance of serum cryptococcal antigen. Clin Infect Dis. 2008;46:e12-8.

22. Baddley JW, Forrest GN; AST Infectious Diseases Community of Practice. Cryptococcosis in solid organ transplantation. Am J Transpl. 2013;13:242-9.

23. Pappas PG, Alexander BD, Andes DR, Hadley S, Kauffman $\mathrm{CA}$, Freifeld $\mathrm{A}$, et al. Invasive fungal infections among organ transplant recipients: results of the TransplantAssociated Infection Surveillance Network (TRANSNET). Clin Infect Dis. 2010;50:1101-11.

24. Cuellar-Rodriguez J, Avery RK, Lard M, Budev M, Gordon SM, Shrestha NK, et al. Histoplasmosis in solid organt transplant recipients: 10 years of experience at a large transplant center in an endemic area. Clin Infect Dis. 2009;49:710-6

25. Batista MV, Pierrotti LC, Abdala E, Clemente WT, Girão ES, Rosa DR, et al. Endemic and opportunistic infections in Brazilian solid organ transplant recipients. Trop Med Int Health. 2011;16:1134-42

26. Martin SI, Fishman JA. AST Infectious Diseases Community of Practice. Pneumocystis pneumonia in solid organ transplantation. Am J Transpl. 2013;13(Suppl 4):272-9 